\title{
Development of brain ventricular system
}

\author{
Vladimir $\operatorname{Korzh}^{1}[$
}

Received: 6 June 2017 / Revised: 20 July 2017 / Accepted: 2 August 2017 / Published online: 5 August 2017

(C) The Author(s) 2017. This article is an open access publication

\begin{abstract}
The brain ventricular system (BVS) consists of brain ventricles and channels connecting ventricles filled with cerebrospinal fluid (CSF). The disturbance of CSF flow has been linked to neurodegenerative disease including hydrocephalus, which manifests itself as an abnormal expansion of BVS. This relatively common developmental disorder has been observed in human and domesticated animals and linked to functional deficiency of various cells lineages facing BVS, including the choroid plexus or ependymal cells that generate CSF or the ciliated cells that cilia beating generates CSF flow. To understand the underlying causes of hydrocephalus, several animal models were developed, including rodents (mice, rat, and hamster) and zebrafish. At another side of a spectrum of BVS anomalies there is the "slit-ventricle" syndrome, which develops due to insufficient inflation of BVS. Recent advances in functional genetics of zebrafish brought to light novel genetic elements involved in development of BVS and circulation of CSF. This review aims to reveal common elements of morphologically different BVS of zebrafish as a typical representative of teleosts and other vertebrates and illustrate useful features of the zebrafish model for studies of BVS. Along this line, recent analyses of the two novel zebrafish mutants affecting different subunits of the potassium voltage-gated channels allowed to emphasize an important functional convergence of the evolutionarily conserved elements of protein transport essential for BVS development, which were revealed by the zebrafish and mouse studies.
\end{abstract}

Vladimir Korzh

vkorzh@iimcb.gov.pl

1 International Institute of Molecular and Cell Biology, Warsaw, Poland
Keywords Brain ventricle - Circumventricular organs . Embryonic cerebrospinal fluid · Hydrocephalus · Voltagegated $\mathrm{K}$ channel $\cdot$ Slit-ventricle syndrome

\section{Introduction}

The brain ventricular system (BVS) consists of brain ventricles and channels that connect ventricles. In mammals the BVS (from anterior to posterior) consists of two lateral (telencephalic) ventricles that via the intraventricular foramina (Monro) connect to the IIIrd (diencephalic) ventricle, which in turn via the sylvius aqueduct connects to the IVth (rhombencephalic or hindbrain) ventricle from where apertures (Lushka and Magendie) connect to subarachnoid space. In other species the BVS is less well understood. Similar to mammals, amphibians and birds have four brain ventricles each of which contains the choroid plexus (CP) $[37,58,80]$.

Traditionally due to absence of means to observe events taking place behind the skull, progress in understanding the BVS relied on indirect or invasive studies of cerebrospinal fluid (CSF) flow. Hence it comes as no surprise that diseases affecting the BVS such as hydrocephalus often result from abnormal circulation of CSF and affect development of brain ventricles [44, 82]. A significant improvement of noninvasive analysis owing to a wider usage of functional magnetic resonance imaging (fMRI) has led to the discovery of intracranial pulsations linked to periodic changes in blood pressure and understanding that CSF flow depends upon systemic circulation. This emphasizes a role of a heart driving circulation, and tissues acting as absorbers of intracranial pulsation [42]. Mechanistically, CSF flow in the developing BVS is driven by a triad of factors-CSF production, beating cilia, and, depending on species, the Reissner fiber (RF) or its soluble components [26, 32, 34, 37, 50, 56, 70]. 
Studies in developmental neurobiology showed that an early developmental abnormality of BVS could be a cause of hydrocephalus and focused attention on ventricular epithelium (ependyma) [22]. Changes of ependymal integrity cause increase of cell proliferation and generation of eCSF resulting in hydrocephalus [9]. At the opposite end, reduction of cell proliferation and/or generation of eCSF may lead to intracranial hypotension and reduction of BVS reminiscent of over-drained ventricular shunt patients (slit-ventricle syndrome; [68] and microcephaly.

Development of BVS has been addressed to some extent. Relatively recent introduction of zebrafish into these studies allowed to study normal development of BVS in real time in vivo with high $3 \mathrm{D}$ resolution and its changes under experimental conditions $[26,32,50,70]$ (Fig. 1a). In contrast, its function during adulthood could be of interest in evolutionary perspective due to major morphogenetic adaptation of aquatic animals during transition to the four-chambered heart, terrestrial habitat and bipedal mode of locomotion, which probably caused major changes in distribution of bodily fluids and blood circulation. Currently, these processes remain not fully understood.
An organization of the neuroepithelial layer lining BVS is of significant interest. By generating CSF and forming a barrier that regulates intracranial pressure, it plays an important role in the development of BVS and its pathology. The specialized functions of the neuroepithelial barrier suggest its specialized organization, including the formation of developmental stage-specific tight junctions containing Claudin5a [3]. In support of this view, during mammalian development the neuroepithelial barrier of ventricular wall develops "strap" junctions limiting transport of biological molecules between BVS and deep cells of neural tube wall. This specialized organization may depend on developmentally restricted expression in ependyma of ECM proteins, namely, Claudin 5 as well as $N$-cadherin, and $\beta$-and $\alpha$-catenins [79].

In view of the fact that studies in model animals revealed a number of genes which deficiency causes early developmental abnormality of BVS, an idea was put forward that the initial phase of formation of this system takes place independent of systemic circulation driven by the cardiac activity, i.e., without involvement of CSF produced by the CP [50, 51, 70, 79] (Fig. 1b). These views could be traced back to pioneering work of Pollay and Curl [66], who demonstrated
Fig. 1 Development of the brain ventricular system of zebrafish. a The dye-filled ventricular system of $48 \mathrm{hpf}$ zebrafish consists of three distinct cavities, which are reshaped as two ventricles and sylvius aqueduct connecting these. b Hypothetical scheme connecting the zebrafish blood circulation and the brain ventricular systems. During development influx of eCSF inflates and shapes the brain ventricular system $[50,51]$. The choroid plexi contribute CSF enriched by ions, micronutrients and proteins. The SCO produces substances of the Reissner fiber, etc. $A$ atrium, $A P$ area postrema, $C C$ central canal, $C P$ choroid plexus, $F$ forebrain, $H$ hindbrain, $M$ midbrain, $S A$ Sylvius aqueduct, $S C O$ subcommissural organ, $V$ heart ventricle, $I I I$ III brain ventricle, $I V$ IV brain ventricle. Black arrow indicates embryonic CSF influx, arrowhead indicates CSF influx, broken line indicates direction of CSF flow. Blue arrow indicates Reissner fiber, red solid lines indicates vasculature and black solid line indicates ependyma
A

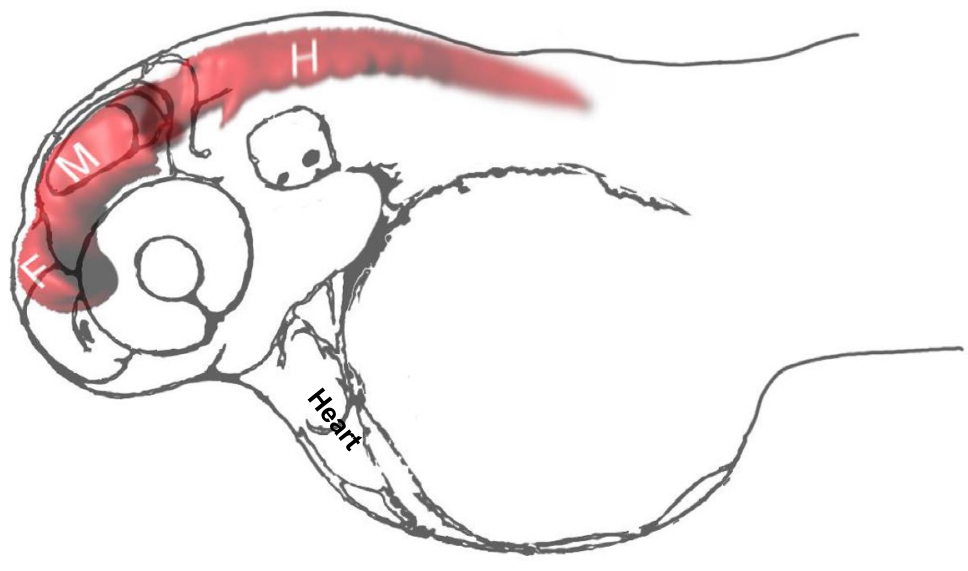

B

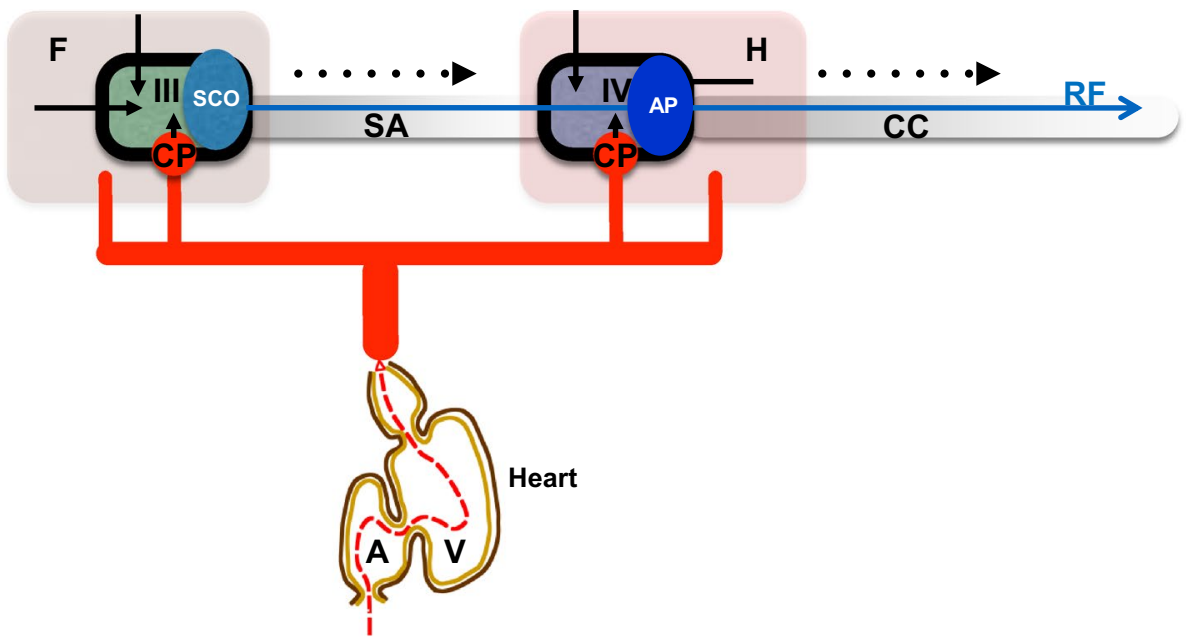


that ependyma generates CSF, and Desmond and Jacobson [23], who, without discriminating effects of eCSF and CSF, suggested that an enlargement of brain cavity in chick embryos requires a temporarily restricted increase of CSF pressure in specific subdivisions of developing brain due to a transient closure of BVS channels. Furthermore, it is accepted that CSF is produced by ependyma as interstitial fluid $[1,18,69]$. At least for now the bioimaging-based analysis of CP development in zebrafish transgenics expressing green fluorescent protein (GFP) led to conclusion that CP is formed after the initial phase of BVS formation [10, 11, 30]. These observations reveal developmental events as demonstrated by accumulation of cytosolic GFP in cells forming the $\mathrm{CP}$ primordium - tela choroidea. It is known that accumulation and maturation of GFP takes at least a couple of hours. Detection of GFP expression in $t$. choroidea depends on timing of initiation of expression of genes tagged by transposon insertion [30] such as, sulf1 encoding Sulfatase 1 , which modulates signaling by heparin-binding growth factors, including FGF2 [20, 47]. Sulf1 deficiency has been linked to microcephaly and cerebral dysfunction [21]. The patterns of GFP expression revealed by conventional confocal microscopy probably illustrate molecular events taking place somewhat earlier. Therefore, it is reasonable to expect that the secretion of CSF may start prior to the detection of GFP in the $t$. choroidea, perhaps, as early as the beginning of BVS inflation. To accurately address this matter, the specific time point of activation of $\mathrm{CP}$ function will need to be re-assessed using much more sensitive and developmental stage-specific bioimaging techniques. Therefore, it is important for the zebrafish BVS anatomy to be characterized in as much detail as that of mammals in order for comparative analysis to become possible.

\section{Neuroanatomy of the brain ventricular system in zebrafish}

Zebrafish (Danio rerio) represents teleosts (bony fish) and belongs to the family Cyprinidae, second largest family of freshwater fish of this planet. Development of its relatively large, semi-transparent, and externally fertilized embryos takes about $52-55 \mathrm{~h}$ at $28.5^{\circ} \mathrm{C}$ outside maternal organism and is easy to monitor under dissecting microscope. These features make the zebrafish embryo a good teaching tool easy to manipulate even in a small laboratory. Given a large collection of transgenics expressing fluorescent proteins in tissue-specific manner and mutants affecting development of various cell lineages, tissues or organs, the zebrafish could be very useful model animal to study development of BVS. Description of BVS in zebrafish is far from being satisfactory. Part of the problem is that comparing to mammals a different number of brain cavities is formed during development and their shape changes rather significantly.
There were attempts to name these according to their position within major subdivisions of brain (telencephalon, diencephalon, midbrain, and hindbrain) [26, 77]. Their connection to brain ventricles is not evaluated with respect to other established landmarks of the brain. The circumventricular organs (CVO) occupy characteristic and well conserved in evolution positions in BVS. In mammals, nine CVOs are commonly recognized: the pineal gland (PIN), subfornical organ (SFO), organum vasculosum laminae terminalis (OVLT), paraventricular organ (PVO), median eminence (ME), neurohypophysis (NH), subcommissural organ (SCO), area postrema (AP) and CP [43]. These organs found mostly along the brain midline in vicinity of the IIIrd and IVth brain ventricles $[25,29,45]$ with exception of CP of lateral ventricles of telencephalon. A vast study described about 18 different CVOs in 31 species belonging to various groups of vertebrates, from cyclostomes to mammals with the NH, ME, SCO and PIN found in almost all vertebrate species examined [75, 76]. In the zebrafish some CVOs were studied, including $\mathrm{CP}$ of the IIIrd and IVth ventricle [10, 11, 30], PIN [55], PP [19], NH and ME [8], SCO [27], AP [53]. Several of CVOs including CP express GFP in zebrafish transgenics ET33-E20 (Gateways; [30, 31].

Unlike the telencephalon in mammals, which develops by inflation, i.e., similar to other brain regions, the zebrafish telencephalon develops by eversion. This means that compared to mammals, there is a significant morphological rearrangement along the mediolateral axis of the telencephalon. Importantly, in teleosts the telencephalon consists of a pair of solid lobes that seem to lack lateral ventricles. Nevertheless, the "telencephalic" ventricle was described in zebrafish $[28,62,81]$. It needs to be mentioned that this discussion was primarily concerned with details of reorganization of neural structures and not the BVS per se. And yet, during embryogenesis, at least three brain cavities develop in the zebrafish brain. This complicates classification of brain ventricles, so it needs to be sorted out based upon additional landmarks. All brain ventricles in mammals are found in specific neuroanatomical location and $\mathrm{CP}$ is a characteristic feature of all ventricles in all species [80]. At the level of forebrain only one $\mathrm{CP}$ was found at the midline of zebrafish brain [10, $11,30]$. This is consistent with an idea that the forebrain CP corresponds to the CPIII found in the diencephalon of other species. The cavity separating the telencephalon and diencephalon has been defined as "telencephalic" ventricle $[28,62]$ is associated with the CPIII, PIN-PP complex, SCO, ME and some other CVOs [31] and, thus, carries features of the IIIrd ventricle of other species. Interestingly, the dye-filling reveals well the dorsal part of the IIIrd ventricle, but not its ventral part [51, 70] (Fig. 1). This could be due to close apposition of the two apical surfaces of ventral brain similar to that in the spinal cord, 
where two apical surfaces closely appose each other dorsally $[46,61]$. More posteriorly, a large transient cavity at the level of midbrain (sometime referred to as optocoele) does not contain $\mathrm{CP}$ or any other CVO. In mammals, the $\mathrm{SCO}$ is located at the posterior border of the IIIrd ventricle close to an entrance into the sylvius aqueduct (SA), which spans the midbrain and midbrain-hindbrain boundary (MHB) to connect to the IVth ventricle. In zebrafish, the SCO is located at the posterior border of IIIrd ventricle at the entrance to optocoele, which connects the IIIrd and IVth ventricles [27]. Thus, the optocoele most probably represents an early phase of the SA development. Similar to the spinal cord, the optocoele contains the midbrain roof plate, which while not being as extended along the dorsal-ventral axis as that of the spinal cord [46] nevertheless is elongated enough to be clearly defined. This illustrates a neuroanatomical element, which is common for the central canal and optocoele. Later in development the optocoele is much reduced due to significant expansion of the optic tectum dorsally and tegmentum ventrally. In parallel, its void acquires an intricate shape, which is more clearly manifested posteriorly [77]. Posterior to the MHB, the roof of hindbrain cavity contains the CPIV $[11,30]$ and the AP at the entrance to the central canal $[31,53]$. These are clear landmarks of the IVth ventricle. Hence as a typical representative of teleosts, the zebrafish contains the BVS, which on one hand is less complex compared to that of mammals, i.e., two ventricles vs. four in mammals (Fig. 2). On the other hand some of its parts such as the IIIrd ventricle and posterior SA acquire rather complex configuration during development although its overall shape is not that different from that in Xenopus.

Given evolutionary conservation of molecular developmental mechanisms, the rules guiding the formation and function of BVS in zebrafish should well represent the basic molecular mechanism of BVS formation and function in mammals at least as far as ventricles III and IV are concerned. This is why the zebrafish is being used currently to study development and genetics of BVS. The zebrafish as a model animal provides a possibility to apply approaches of direct genetics, such as medium- to large-scale mutagenesis screens and bioimaging of the developing BVS in vivo. Still, it is possible that due to significant evolutionary distance between teleosts and mammals, some genes and/or gene functions with a role in the BVS development in teleosts were acquired due to an extra round of genome duplication in teleosts or lost or modified significantly during evolution of land animals and transition from aquatic environment to a terrestrial one, or in evolution of human during transition to bipedal mode of locomotion and vertical body posture. Hence more studies are requires to address developmental and evolutional diversity of the molecular machinery driving formation and function of BVS in zebrafish.
A

B

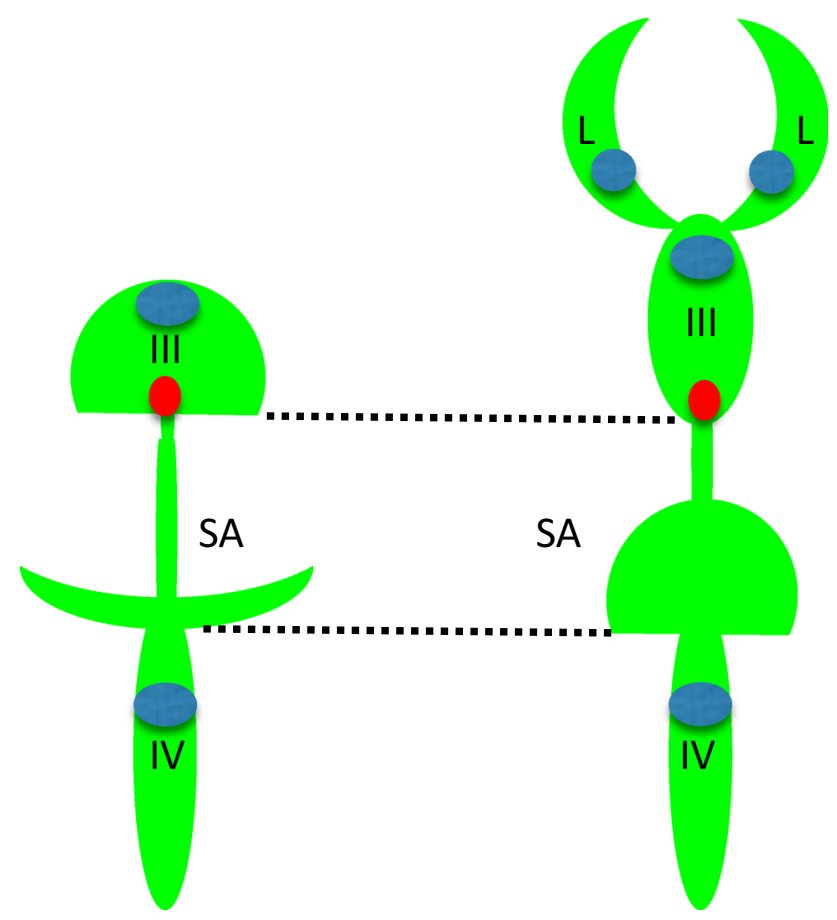

Fig. 2 Comparison of brain ventricular system in $6 \mathrm{dpf}$ zebrafish (a) and stage 53 Xenopus (b). The zebrafish larval BVS contains two ventricles unlike that of larval Xenopus. Whereas the IIIrd ventricle and posterior SA by the end of embryogenesis acquire rather complex configuration these are not that different from corresponding part of BVS in Xenopus. Green-ventricular system, blue - choroid plexus, red-subcommissural organ, $I I I$-IIIrd ventricle, $I V$-IVth ventricle, $L$-lateral ventricle, $S A$-Sylvius aqueduct (modified from Turner et al. [77], and Mogi et al. [58], correspondingly)

\section{Developmental genetics of the brain ventricular system}

At the end of 2016 the term "abnormal brain ventricular system morphology" was associated with 549 mice mutants (http://www.informatics.jax.org/searchtool/Search.do?que ry =brain+ventricle \&submit=Quick+Search) with 183 mutants showing enlarged brain ventricles and only ten with reduced brain ventricles. This suggested that the diagnosis of BVS deficiencies still largely relies on detection of gross morphological abnormality. Perhaps, it is easier to detect hydrocephalus compared to reduction of brain ventricles. Along the same line, given a connection of the reduction of brain ventricles and microcephaly and the fact that a similar search for the term "microcephaly" brought about 143 entries, a number of mutants with reduced brain ventricles could be underestimated. To add to that, hydrocephalus might be caused by defects in evolutionary conserved (in teleosts and mammals) components of signaling pathways, in particular those involved in planar cell polarity and ciliogenesis $[65,73]$. Not all ependymal cells are ciliated and at least in mammals a significant proportion of these are 
tanycytes expressing a variety of tight junction proteins [2, $48,60,71]$. This makes it possible that defects of BVS are intricately linked to defects of tissue integrity.

Despite limitations of current knowledge brought about by technical challenges, experimental evidence obtained due to analysis of zebrafish mutants occasionally allows to combine results obtained in different areas of science and develop a novel perspective. This concerns the formation of BVS also. During the large-scale mutagenesis screens of the 1990 s, several mutants with deficient BVS, including nagie oko (nok), which affects mpp5a encoding the membrane protein, palmitoylated 5a (MAGUK p55 subfamily member 5 $[54,78]$, and snakehead (snk), which affects atplala.1 [40, 51] were found. It took several years to map these mutants and reveal their true potential for studies of the developing BVS. The nok mutants are characterized by abnormal brain neuroepithelium with no clear midline and disrupted junctional protein expression. They fail to undergo ventricle morphogenesis in a process dependent on their interaction of Mpp5a with Crumbs2a (Crb2a), which failure affects the transport of proteins of the apical complex [12, 17, 51]. Similar phenotype was described in the pard6 $\gamma \beta$ mutant [46, $61,63]$. In contrast, analysis of snk mutants with impaired BVS inflation revealed a complex role of Atp $1 \alpha 1$ a during formation of cohesive neuroepithelium, restriction of neuroepithelial permeability and production of CSF $[17,51]$.

This led to an understanding that a systematic classification of collection of zebrafish mutants exhibiting ventricle deficiency assayed using ventricular dye-filling, bioimaging and immunohistochemistry could be instrumental in defining the different stages of BVS development. As a result, four phenotypically distinct classes of mutants with early to late developmental defects ranging from abnormalities of epithelial junction and ventricle expansion to defects of MHB and ventricle lumen expansion were defined [50]. For example, detailed analysis of mutants with deficient MHB demonstrated that its formation is not entirely dependent upon changes in intracranial pressure. Here the two phases of this process were described. First includes a significant shortening of the MHB cells followed by the laminin-dependent basal constriction and apical expansion of a specialized group of MHB cells [35]. Second includes epithelial relaxation involving the myosin phosphatase regulator Mypt1 that regulates changes in cell shape during stretching of neuroepithelium. It is required for expansion of ventricular lumen [36]. Thus, formation of BVS is based upon a complex network of evolutionary conserved molecular events that could be efficiently analyzed using zebrafish mutants.

Despite being instrumental, mutants derived from the large-scale chemical mutagenesis were not easy to map. Therefore, there is a group of unmapped mutants relevant for studies of BVS, whose analysis is limited by the lack of knowledge of genes affected-zon, all, fun [4, 49, 82]. This situation may change as mapping of mutants is getting less challenging. Similarly, genes and/or mutants (e.g., Table 1) will be re-evaluated in future studies using other functional assays and their classification may change. There is also a group of insertional mutants that cause hydrocephalus.

Table 1 Some zebrafish mutants with defects in the brain ventricular system

\begin{tabular}{|c|c|c|c|c|}
\hline Gene & Zebrafish mutant/phenotype & Mice phenotype & Human phenotype & References \\
\hline mpp5a & nok/reduced ventricle & No & Kabuki syndrome (microcephaly) & $\begin{array}{l}{[12,51] ; \text { http://www.malacards.org/search/ }} \\
\text { results/mpp5 }\end{array}$ \\
\hline prkci & has/reduced ventricle & No & Huntington disease & $\begin{array}{l}\text { [50]; http://www.malacards.org/search/results/ } \\
\text { prkci }\end{array}$ \\
\hline$c r b 2$ & ome/reduced ventricle & Open neural tube & Ventriculomegaly & $\begin{array}{l}\text { [50]; http://www.malacards.org/search/results/ } \\
\text { crb2 }\end{array}$ \\
\hline $\operatorname{pard} 6 \gamma \beta$ & pard $6 \gamma \beta /$ reduced lumen & NA & NA & $\begin{array}{l}\text { [46, 61]; http://omim.org/ } \\
\text { entry/608976?search=pard6g\%20 } \\
\text { gene\&highlight=pard6g\%20gene }\end{array}$ \\
\hline med12 & ott/reduced ventricle & NA & Craniorachischisis, exencephaly & $\begin{array}{l}\text { [50]; http://www.malacards.org/search/results/ } \\
\text { med12 }\end{array}$ \\
\hline med14 & $\log /$ reduced ventricle & NA & NA & $\begin{array}{l}\text { http://omim.org/entry/300182? search=med } 14 \& \\
\text { highlight=med14 }\end{array}$ \\
\hline lamc1 & sly/reduced ventricle & E5.5 lethal & Occipital cephalocoele (?) & [50]; OMIM*150290 \\
\hline lamb1 & gap/reduced ventricle & Embryonic lethal & Hydrocephalus & [50]; [67] \\
\hline atplal & $s n k /$ reduced ventricle & Hypertension & & {$[17,51] ;$ OMIM*182310 } \\
\hline kcnbl & kcnbl/reduced ventricle & NA & Epileptic encephalopathy & {$[70,72] ;$ OMIM*600397 } \\
\hline$f n 1$ & nat/reduced ventricle & Neuronal apoptosis & & {$[50]$} \\
\hline$s f p q$ & wis/reduced ventricle & Renal dysfunction & NA & [50]; OMIM*605199 \\
\hline$k c n g 4 b$ & kcng $4 b /$ hydrocephalus & NA & NA & [70] \\
\hline
\end{tabular}


While these were mapped, they remain characterized insufficiently (rps29, rpl7, rps12, sox32, etc. [5]). Some of these mutants were linked to genes with a role in cell death or some other developmental events, which may not be relevant in the specific context of BVS development, whereas those linked to regulation in blood circulation could be rather useful. For these events to be better understood, further studies of such mutants are required. The availability of these research tools ready to be analyzed means that an entry threshold into this field is relatively low.

Some results obtained using methods of transient block and/or inhibition of gene activity probably illustrates incomplete suppression of gene activity and/or peculiarity of experimental procedure involved. For example, the targeted morpholino-mediated knockdown of either Atp1 $\alpha 3 \mathrm{a}$ or Atp $1 \alpha 3 b$ leads to hydrocephalus, which authors interpreted as a result of imbalance of ion transport across plasma membrane resulting in excessive accumulation of CSF in the BVS [24]. In contrast, the analysis of the atpla3a $a^{\text {tpllo }}$ gene trap mutant zebrafish failed to show hydrocephalus in atpla $3^{\text {atpllo }}$ homozygotes, but demonstrated larval lethality of atpla $3 a^{\text {tpllo }}$ homozygotes. This was interpreted in favor of an essential role for Atp1 $\alpha 3 \mathrm{a}$ in neural development and/ or physiology [7], i.e., in concert with postembryonic lethality observed in the atpla3 mutant mice [59]. This example raises awareness that interpretation of results of the transient loss-of-function analysis should be done with caution. And yet one should keep in mind that a complete loss-of-function of gene activity may be a rare event in human population, where mutations detected could be, therefore, much less disruptive. Hence it is possible that the phenotype observed upon transient loss-of-function may to some extent represent the effect of more deleterious mutations. Alternatively, it is also possible that the effect of such experimental procedure is unspecific. These situations will need more experimental evidence to be analyzed before some of controversies could be resolved. At least for now a bulk of data links ATP $1 \alpha 3$ to a range of neurological disorders, but fails to support brain ventricle dilation observed in zebrafish (http://omim.org/ entry/182350).

Here it would be appropriate to remind about a large cohort of studies of the popular rodent model of hydrocephalus-hyh mutant mice $[15,41]$ and compare that with recent data obtained using the zebrafish model. In the latter a study of a role of the Kcnb1-Keng4b axis in the BVS development revealed a rather satisfactory correlation of the mutant (stable) and morphant (transient) phenotypes as well as dominant-positive and-negative studies [70]. It was shown in mice that the BVS development is affected by the molecular impairment of the SNARE-mediated protein transport essential for regulated exocytosis of neurotransmitters, apical localization of proteins and determination of neuroepithelial cell fate. $\alpha$-Snap is involved in a wide variety of membrane fusion events in eukaryotic cells, including the disassembly of the cis-SNARE complex and the target compartment, i.e., recycling and retrograde transport of components of $v$-SNAREs [39]. In the hyh mice a mutation of $\alpha$-Snap results in delamination of neuroepithelial cells lining the BVS followed by hydrocephalus [16, 38]. In developing mice brain, proliferating cells in the ventricular zone (VZ) are found in two distinct regions: the dorsal walls of the IIIrd ventricle and SA. In hydrocephalic hyh mutants, the dorsal walls of the IIIrd ventricle and SA expand enormously, probably partially due to increased ependymogenesis [9]. A possibility exists that such increase of cell proliferation compensates for delamination of cells taking place earlier.

Such scenario is very similar to that described recently in the zebrafish insertional mutant with aberrant splicing of the kcng $4 b$ mRNA, which encodes the silent subunit of the voltage-gated $\mathrm{Kv}$ channel-Keng4 (or Kv6.4; [70]). It was shown by electrophysiologists that Kv6.4 functionally antagonizes electrically active subunit of the voltage-gated Kv channel-Kv2.1 (or Kcnb1; [13, 14, 64]). This mutation in Kcng $4 \mathrm{~b}$ may cause the dominant-negative peptide to be formed such as the one used to block the activity of $\mathrm{Kv}$ channel in vitro [6]. Unlike its homologue kcng $4 a$, which is expressed in sensory cells, kcng $4 b$ is expressed in epithelial cells lining the cavities of BVS and other hollow organs, including otic vesicles as well as anterior and posterior eye chambers and lens. Similar to that in hyh mutants, when causing hydrocephalus $k c n g 4 b$ mutation first causes delamination of ependymal cells followed by their excessive proliferation. Similarly, events uncovered in this case could be traced to aberrant protein transport. To reach the plasma membrane, Kv6.4 interacts with Kv2.1, which transports Kv6.4 from ER to plasma membrane in a process of SNARE-mediated protein transport [14, 64]. Since developmental defects described for the hyh mice and Kcng $4 \mathrm{~b}$ (Kv6.4)-deficient zebrafish are very similar, it might be not too surprising that one of the outcomes of hyh mutation could be a deficient function of $\mathrm{Kv}$ channels due to abnormal protein transport. Indeed, it was shown already that one of $v$-SNAREs-VAMP2, interacts with Kv2.1 and inactivates it [52], whereas components of $t$-SNAREs-Syntaxin $1 \mathrm{~A}$ and the Syntaxin/SNAP-25 complex bind directly to the Kv2.1 channel C-terminus [57, 74]. A disturbance of protein transport in hyh mutants with all probability will impact that of Kv2.1, which may explain the remarkable phenotypical similarity of phenotypes of the zebrafish and mice mutants (Fig. 3).

Taking into account counteracting activities of Kv6.4 and Kv2.1 established by electrophysiologists in vitro, it was gratifying to see that a mutation of $k c n b l$ generated by site-specific mutagenesis caused a developmental phenotype reverse comparing to that of $k c n g 4 b$, i.e., reduced cell proliferation and reduced BVS. Further support for functional 


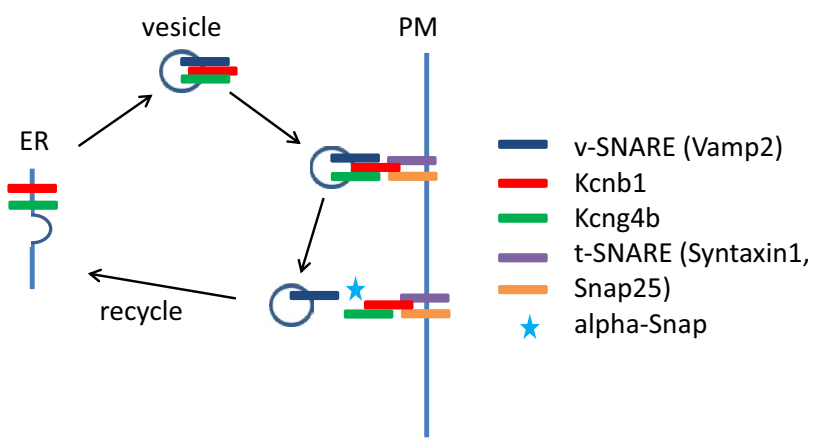

Fig. 3 Genetic analyses reveal a role of the Kcnb1-Kcng4 axis in development of the BVS. Mutation/LOF of Kcg $4 \mathrm{~b}$ causes strong hydrocephalus and GOF-almost complete failure of ventricle inflation. In contrast, Kcnb1 mutant shows the slit-ventricle phenotype. The defect of Kcnb1 not only eliminates function of this protein as an electrically active subunit of voltage-gated $\mathrm{Kv}$ channel. Given its role in transport of Kcng4b, the defect of Kcnb1 negatively impacts function of both proteins, which counteract each other activity at the plasma membrane. Thus, the complete LOF of Kcnb1 could be partially compensated by a deficiency of Kcng4b transport. Note that Keng $4 \mathrm{~b}$ is not expressed in adult animals suggesting that its modulation of Kcnb1 activity takes place only during development

antagonism of the two subunits of the Kv channel during BVS development was provided when over-expression of these two genes caused the phenotypes reverse to those in mutants [70]. Intriguingly, mutations of KCNB1 in human were linked to early infantile epileptic encephalopathy (OMIM*600397, OMIM\#616056). Hence these findings revealed a common molecular denominator for two human hereditary diseases-epileptic encephalopathy and hydrocephalus. More broadly, it suggested that different mutations of KCNB1 may cause a wide range of hereditary diseases-a notion which certainly needs to be explored further. Recently, the epilepsy phenotype has been characterized in the zebrafish mutant of the Syntaxin-binding protein 1 (Stxbp1; [33]). Taken together, genetic and biochemical evidences available illustrate the functional link between the components of protein transport and BVS development in model animals and human.

\section{Conclusion}

The zebrafish has emerged as a good animal model to study BVS development and various aspects of human hereditary diseases affecting BVS such as the slit-ventricle syndrome and hydrocephalus. Analysis of mice and zebrafish mutants provided different, but complementary views on a role of protein transport in physiology of ependymal cells lining fluid-filled organs such as brain ventricles. In view of expression of the same proteins in cells lining internal cavities of the ear and eye, it is expected that future studies will reveal that similar mechanisms dependent on voltage-gated $\mathrm{K}$ channel and interplay of its subunits operate in these organs too. This information is important for understanding molecular mechanisms of development of the fluid-filled organs and, in particular, integrity of neuroepithelial lining as well as pathology caused by its loss.

Acknowledgements $\mathrm{He}$ is thankful to Dr. Cecilia Winata for critical comments and personnel of the fish facilities of the IMCB (Singapore) and IIMCB (Warsaw) for fish maintenance. VK is supported by a Grant for visiting professor at the IIMCB in Warsaw and the Opus Grant (2016/21/B/NZ3/00354) from the NCN, Poland.

\section{Compliance with ethical standards}

Conflict of interest VK declares no conflict of interest.

Open Access This article is distributed under the terms of the Creative Commons Attribution 4.0 International License (http://creativecommons.org/licenses/by/4.0/), which permits unrestricted use, distribution, and reproduction in any medium, provided you give appropriate credit to the original author(s) and the source, provide a link to the Creative Commons license, and indicate if changes were made.

\section{References}

1. Abbott NJ (2004) Evidence for bulk flow of brain interstitial fluid: significance for physiology and pathology. Neurochem Int 45:545-552. doi:10.1016/j.neuint.2003.11.006

2. Abbott NJ, Patabendige AAK, Dolman DEM et al (2010) Structure and function of the blood-brain barrier. Neurobiol Dis 37:13-25. doi:10.1016/j.nbd.2009.07.030

3. Abdelilah-Seyfried S (2010) Claudin-5a in developing zebrafish brain barriers: another brick in the wall. BioEssays 32:768-776. doi:10.1002/bies.201000045

4. Abdelilah S, Mountcastle-Shah E, Harvey M et al (1996) Mutations affecting neural survival in the zebrafish Danio rerio. Development 123:217-227

5. Amsterdam A, Nissen RM, Sun Z et al (2004) Identification of 315 genes essential for early zebrafish development. Proc Natl Acad Sci USA 101:12792-12797. doi:10.1073/ pnas.0403929101

6. Babila T, Moscucci A, Wang $\mathrm{H}$ et al (1994) Assembly of mammalian voltage-gated potassium channels: evidence for an important role of the first transmembrane segment. Neuron 12:615-626 (published erratum appears in Neuron 1996 May; 16(5):following 1060)

7. Balciuniene J, Nagelberg D, Walsh KT et al (2013) Efficient disruption of zebrafish genes using a Gal4-containing gene trap. BMC Genom 14:1. doi:10.1186/1471-2164-14-619

8. Bassi I, André V, Marelli F et al (2016) The zebrafish: an emerging animal model for investigating the hypothalamic regulation of reproduction. Minerva Endocrinol 41:250-265

9. Bátiz LF, Jiménez AJ, Guerra M et al (2011) New ependymal cells are born postnatally in two discrete regions of the mouse brain and support ventricular enlargement in hydrocephalus. Acta Neuropathol 121:721-735. doi:10.1007/s00401-011-0799-x

10. Bill B, Korzh V (2014) Choroid plexus in developmental and evolutionary perspective. Front Neurosci 8:1-11. doi:10.3389/ fnins.2014.00363 
11. Bill BR, Balciunas D, McCarra JA et al (2008) Development and notch signaling requirements of the zebrafish choroid plexus. PLoS One 3:1-9. doi:10.1371/journal.pone.0003114

12. Bit-Avragim N, Hellwig N, Rudolph F et al (2008) Divergent polarization mechanisms during vertebrate epithelial development mediated by the Crumbs complex protein Nagie oko. J Cell Sci 121:2503-2510. doi:10.1242/jcs.033167

13. Bocksteins E, Labro AJ, Mayeur E et al (2009) Conserved negative charges in the $\mathrm{N}$-terminal tetramerization domain mediate efficient assembly of Kv2.1 and Kv2.1/Kv6.4 channels. J Biol Chem 284:31625-31634. doi:10.1074/jbc.M109.039479

14. Bocksteins E, Snyders DJ (2012) Electrically silent Kv subunits: their molecular and functional characteristics. Physiology 27:7384. doi:10.1152/physiol.00023.2011

15. Bronson R, Lane P (1990) Hydrocephalus with hop gait (hyh): a new mutation on chromosome 7 in the mouse. Brain Res Dev Brain Res 54:131-136

16. Chae TH, Kim S, Marz KE et al (2004) The hyh mutation uncovers roles for alpha Snap in apical protein localization and control of neural cell fate. Nat Genet 36:264-270. doi:10.1038/ng1302

17. Chang JT, Lowery LA, Sive H (2012) Multiple roles for the $\mathrm{Na}$, K-ATPase subunits, Atp1a1 and Fxyd1, during brain ventricle development. Dev Biol 368:312-322. doi:10.1016/j. ydbio.2012.05.034

18. Chikly B, Quaghebeur J (2013) Reassessing cerebrospinal fluid (CSF) hydrodynamics: a literature review presenting a novel hypothesis for CSF physiology. J Bodyw Mov Ther 17:344-354. doi:10.1016/j.jbmt.2013.02.002

19. Concha ML, Wilson SW (2001) Asymmetry in the epithalamus of vertebrates. J Anat 199:63-84. doi:10.1046/j.1469-7580.2001.19910063.x

20. Dai Y, Yang Y, MacLeod V et al (2005) HSulf-1 and HSulf-2 are potent inhibitors of myeloma tumor growth in vivo. J Biol Chem 280:40066-40073. doi:10.1074/jbc.M508136200

21. Day-Salvatore D, McLean D (1998) Blepharophimosis, hypoplastic radius, hypoplastic left heart, telecanthus, hydronephrosis, fused metacarpals, and "prehensile" halluces: a new syndrome? Am J Med Genet 80:309-313. doi:10.1002/ (SICI)1096-8628(19981204)80:4<309:AID-AJMG2>3.0.CO;2-J

22. Del Bigio MR (2010) Neuropathology and structural changes in hydrocephalus. Dev Disabil Res Rev 16:16-22. doi:10.1002/ ddrr.94

23. Desmond ME, Jacobson AG (1977) Embryonic brain enlargement requires cerebrospinal fluid pressure. Dev Biol 57:188-198. doi:10.1016/0012-1606(77)90364-5

24. Doğanli C, Beck HC, Ribera AB et al (2013) $\alpha 3 \mathrm{Na}^{+} / \mathrm{K}^{+}$-ATPase deficiency causes brain ventricle dilation and abrupt embryonic motility in zebrafish. J Biol Chem 288:8862-8874. doi:10.1074/ jbc.M112.421529

25. Duvernoy HM, Risold PY (2007) The circumventricular organs: an atlas of comparative anatomy and vascularization. Brain Res Rev 56:119-147

26. Fame RM, Chang JT, Hong A et al (2016) Directional cerebrospinal fluid movement between brain ventricles in larval zebrafish. Fluids Barriers CNS 13:11. doi:10.1186/s12987-016-0036-z

27. Fernández-Llebrez $\mathrm{P}$, Miranda E, Estivill-Torrús $\mathrm{G}$ et al (2001) Analysis and quantification of the secretory products of the subcommissural organ by use of monoclonal antibodies. Microsc Res Tech 52:510-519. doi:10.1002/10970029(20010301)52:5<510:AID-JEMT1036>3.0.CO;2-A

28. Folgueira M, Bayley P, Navratilova P et al (2012) Morphogenesis underlying the development of the everted teleost telencephalon. Neural Dev 7:32. doi:10.1186/1749-8104-7-32

29. Ganong WF (2000) Circumventricular organs: definition and role in the regulation of endocrine and autonomic function. Clin Exp Pharmacol Physiol 27:422-427. doi:10.1046/j.1440-1681.2000.03259.x

30. García-Lecea M, Kondrychyn I, Fong SH et al (2008) In vivo analysis of choroid plexus morphogenesis in zebrafish. PLoS One 3:1. doi:10.1371/journal.pone.0003090

31. García-Lecea M, Kondrychyn I, Teh C et al Development of circumventricular organs in the mirror of zebrafish enhancer-trap transgenics (in preparation)

32. Grimes DT, Boswell CW, Morante NFC et al (2016) Zebrafish models of idiopathic scoliosis link cerebrospinal fluid flow defects to spine curvature. Science 80(1284):1281-1284. doi:10.1126/ science.aaf6419

33. Grone BP, Marchese M, Hamling KR et al (2016) Epilepsy, behavioral abnormalities, and physiological comorbidities in syntaxin-binding protein 1 (STXBP1) mutant zebrafish. PLoS One. doi:10.1371/journal.pone.0151148

34. Guerra MM, González C, Caprile T et al (2015) Understanding how the subcommissural organ and other periventricular secretory structures contribute via the cerebrospinal fluid to neurogenesis. Front Cell Neurosci 9:480. doi:10.3389/fncel.2015.00480

35. Gutzman JH, Graeden EG, Lowery LA et al (2008) Formation of the zebrafish midbrain-hindbrain boundary constriction requires laminin-dependent basal constriction. Mech Dev 125:974-983. doi:10.1016/j.mod.2008.07.004

36. Gutzman JH, Sive H (2010) Epithelial relaxation mediated by the myosin phosphatase regulator Mypt 1 is required for brain ventricle lumen expansion and hindbrain morphogenesis. Development 137:795-804. doi:10.1242/dev.042705

37. Hagenlocher C, Walentek P, Muller C et al (2013) Ciliogenesis and cerebrospinal fluid flow in the developing Xenopus brain are regulated by foxj1. Cilia 2:12. doi:10.1186/2046-2530-2-12

38. Hong H-K, Chakravarti A, Takahashi JS (2004) The gene for soluble $N$-ethylmaleimide sensitive factor attachment protein alpha is mutated in hydrocephaly with hop gait (hyh) mice. Proc Natl Acad Sci USA 101:1748-1753. doi:10.1073/pnas.0308268100

39. Hong W (2005) SNAREs and traffic. Biochim Biophys Acta Mol Cell Res 1744:120-144. doi:10.1016/j.bbamcr.2005.03.014

40. Jiang YJ, Brand M, Heisenberg CP et al (1996) Mutations affecting neurogenesis and brain morphology in the zebrafish, Danio rerio. Development 123:205-216

41. Jiménez AJ, Rodríguez-Pérez LM, Domínguez-Pinos MD et al (2014) Increased levels of tumour necrosis factor alpha (TNF??) but not transforming growth factor-beta 1 (TGF??1) are associated with the severity of congenital hydrocephalus in the hyh mouse. Neuropathol Appl Neurobiol 40:911-932. doi:10.1111/nan.12115

42. Johanson CE, Duncan JA, Klinge PM et al (2008) Multiplicity of cerebrospinal fluid functions: new challenges in health and disease. Cerebrospinal Fluid Res 5:10. doi:10.1186/1743-8454-5-10

43. Joly JS, Osório J, Alunni A et al (2007) Windows of the brain: towards a developmental biology of circumventricular and other neurohemal organs. Semin Cell Dev Biol 18:512-524. doi:10.1016/j.semcdb.2007.06.001

44. Kahle KT, Kulkarni AV, Limbrick DD, Warf BC (2016) Hydrocephalus in children. Lancet 387:788-799. doi:10.1016/ S0140-6736(15)60694-8

45. Kaur C, Ling E-A (2017) The circumventricular organs. Histol Histopathol 11881. doi: 10.14670/HH-11-881

46. Kondrychyn I, Teh C, Sin M, Korzh V (2013) Stretching morphogenesis of the roof plate and formation of the central canal. PLoS One 8:1-12. doi:10.1371/journal.pone.0056219

47. Lai J, Chien J, Staub J et al (2003) Loss of HSulf-1 up-regulates heparin-binding growth factor signaling in cancer. J Biol Chem 278:23107-23117. doi:10.1074/jbc.M302203200

48. Langlet F, Mullier A, Bouret SG et al (2013) Tanycyte-like cells form a blood-cerebrospinal fluid barrier in the circumventricular 
organs of the mouse brain. J Comp Neurol 521:3389-3405. doi:10.1002/cne.23355

49. Lowery LA, De Rienzo G, Gutzman JH, Sive H (2009) Characterization and classification of zebrafish brain morphology mutants. Anat Rec 292:94-106. doi:10.1002/ar.20768

50. Lowery LA, Sive H (2009) Totally tubular: the mystery behind function and origin of the brain ventricular system. BioEssays 31:446-458. doi:10.1002/bies.200800207

51. Lowery LA, Sive H (2005) Initial formation of zebrafish brain ventricles occurs independently of circulation and requires the nagie oko and snakehead/atp1a1a.1 gene products. Development 132:2057-2067. doi:10.1242/dev.01791

52. Lvov A, Greitzer D, Berlin S et al (2009) Rearrangements in the relative orientation of cytoplasmic domains induced by a membrane-anchored protein mediate modulations in $\mathrm{Kv}$ channel gating. J Biol Chem 284:28276-28291. doi:10.1074/jbc. M109.028761

53. Ma PKM (1997) Catecholaminergic systems in the zebrafish. III. Organization and projection pattern of medullary dopaminergic and noradrenergic neurons. J Comp Neurol 381:411-427. doi:10.1002/ (SICI)1096-9861(19970519)381:4<411:AID-CNE2>3.0.CO;2-5

54. Malicki J, Neuhauss SC, Schier AF et al (1996) Mutations affecting development of the zebrafish retina. Development 123:263273. doi:10.5167/uzh-229

55. Masai I, Heisenberg CP, Barth KA et al (1997) Floating head and masterblind regulate neuronal patterning in the roof of the forebrain. Neuron 18:43-57. doi:10.1016/S0896-6273(01)80045-3

56. Meiniel A (2007) The secretory ependymal cells of the subcommissural organ: which role in hydrocephalus? Int J Biochem Cell Biol 39:463-468. doi:10.1016/j.biocel.2006.10.021

57. Michaelevski I, Chikvashvili D, Tsuk S et al (2003) Direct interaction of target SNAREs with the Kv2.1 channel: modal regulation of channel activation and inactivation gating. J Biol Chem 278:34320-34330. doi:10.1074/jbc.M304943200

58. Mogi K, Adachi T, Izumi S, Toyoizumi R (2012) Visualisation of cerebrospinal fluid flow patterns in albino Xenopus larvae in vivo. Fluids Barriers CNS 9:9. doi:10.1186/2045-8118-9-9

59. Moseley AE, Williams MT, Schaefer TL et al (2007) Deficiency in $\mathrm{Na}$, K-ATPase $\alpha$ isoform genes alters spatial learning. Motor Act Anxiety Mice 27:616-626. doi:10.1523/ JNEUROSCI.4464-06.2007

60. Mullier A, Bouret S, Prevot V et al (2010) Differential distribution of tight junction proteins suggests a role for tanycytes in bloodhypothalamus barrier regulation in the adult mouse brain. J Comp Neurol 518:943-962. doi:10.1002/cne.22273

61. Munson C, Huisken J, Bit-Avragim N et al (2008) Regulation of neurocoel morphogenesis by Pard6 $\gamma$ b. Dev Biol 324:41-54. doi:10.1016/j.ydbio.2008.08.033

62. Nieuwenhuys R (2011) The development and general morphology of the telencephalon of actinopterygian fishes: synopsis, documentation and commentary. Brain Struct Funct 215:141-157. doi:10.1007/s00429-010-0285-6

63. Ober EA, Verkade H, Field HA, Stainier DYR (2006) Mesodermal Wnt $2 \mathrm{~b}$ signalling positively regulates liver specification. Nature 442:688-691. doi:10.1038/nature04888

64. Ottschytsch N, Raes A, Van Hoorick D, Snyders DJ (2002) Obligatory heterotetramerization of three previously uncharacterized Kv channel alpha-subunits identified in the human genome. Proc Natl Acad Sci USA 99:7986-7991. doi:10.1073/pnas.122617999

65. Pathak NH, Drummond IA (2009) Polyglutamylation and the fleer gene. Methods Cell Biol 94:317-332. doi:10.1016/ S0091-679X(08)94016-4
66. Pollay M, Curl F (1967) Secretion of cerebrospinal fluid by the ventricular ependyma of the rabbit. Am J Physiol 213:1031-1038 (PMID: 6051171)

67. Radmanesh F, Caglayan A, Silhavy J et al (2013) Mutations in LAMB1 cause cobblestone brain malformation without muscular or ocular abnormalities. Am J Hum Genet 92:468-474. doi:10.1016/j.ajhg.2013.02.005

68. Rekate HL (2008) Shunt-related headaches: The slit ventricle syndromes. Child's Nerv Syst 24:423-430. doi:10.1007/ s00381-008-0579-7

69. Sakka L, Coll G, Chazal J (2011) Anatomy and physiology of cerebrospinal fluid. Eur Ann Otorhinolaryngol Head Neck Dis 128:309-316. doi:10.1016/j.anorl.2011.03.002

70. Shen H, Bocksteins E, Kondrychyn I et al (2016) Functional antagonism of voltage-gated $\mathrm{K}^{+}$channel $\alpha$-subunits in the developing brain ventricular system. Development 143:4249-4260. doi:10.1242/dev.140467

71. Szathmari A, Champier J, Ghersi-Egea JF et al (2013) Molecular characterization of circumventricular organs and third ventricle ependyma in the rat: potential markers for periventricular tumors. Neuropathology 33:17-29. doi:10.1111/j.1440-1789.2012.01321.x

72. Torkamani A, Bersell K, Jorge B et al (2014) De novo KCNB1 mutations in epileptic encephalopathy. Ann Neurol 76:529-540. doi:10.1002/ana.24263

73. Treat AC, Wheeler DS, Stolz DB et al (2016) The PDZ protein $\mathrm{Na}^{+} / \mathrm{H}^{+}$exchanger regulatory factor-1 (NHERF1) regulates planar cell polarity and motile cilia organization. PLoS One 11:1-22. doi:10.1371/journal.pone.0153144

74. Tsuk S, Lvov A, Michaelevski I et al (2008) Formation of the full SNARE complex eliminates interactions of its individual protein components with the Kv2.1 channel. Biochemistry 47:8342-8349. doi:10.1021/bi800512p

75. Tsuneki K (1986) A survey of occurrence of about seventeen circumventricular organs in brains of various vertebrates with special reference to lower groups. J Hirnforsch 27:441-470

76. Tsuneki K (1987) A histological survey on the development of circumventricular organs in various vertebrates: developmental biology. Zoolog Sci 4:497-521

77. Turner MH, Ullmann JFP, Kay AR (2012) A method for detecting molecular transport within the cerebral ventricles of live zebrafish (Danio rerio) larvae. J Physiol 590:2233-2240. doi:10.1113/ jphysiol.2011.225896

78. Wei X, Malicki J (2002) Nagie oko, encoding a MAGUK-family protein, is essential for cellular patterning of the retina. Nat Genet 31:150-157. doi:10.1038/ng883

79. Whish S, Dziegielewska KM, Møllgård K et al (2015) The inner csf-brain barrier: developmentally controlled access to the brain via intercellular junctions. Front Neurosci 9:1-15. doi:10.3389/ fnins.2015.00016

80. Wilting J, Christ B (1989) An experimental and ultrastructural study on the development of the avian choroid plexus. Cell Tissue Res 255:487-494. doi:10.1007/BF00218783

81. Wullimann MF (2009) Secondary neurogenesis and telencephalic organization in zebrafish and mice: a brief review. Integr Zool 4:123-133

82. Zhang J, Williams MA, Rigamonti D (2006) Genetics of human hydrocephalus. J Neurol 253:1255-1266. doi:10.1007/ s00415-006-0245-5 